\title{
In Vitro Generation of Human Cytomegalovirus pp65 Antigenemia, Viremia, and LeukoDNAemia
}

\author{
Maria Grazia Revello, ${ }^{\star}$ Elena Percivalle,, Eloisa Arbustini,, Ruggero Pardi,§ Silvano Sozzani," and Giuseppe Gerna* \\ *Servizio di Virologia, and ${ }^{\ddagger}$ Istituto di Anatomia Patologica, Istituto di Ricovero e Curd a Carattere Scientifico Policlinico San Matteo, \\ 27100 Pavia, Italy; ${ }^{\S}$ Dipartimento di Biotecnologie, Istituto di Ricovero e Curd a Carattere Scientifico San Raffaele, Università di Milano,

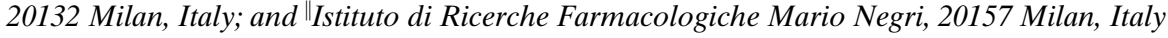

\begin{abstract}
Immunocompromised patients with disseminated human cytomegalovirus (HCMV) infection have circulating PMN carrying HCMV pp65 (antigenemia), infectious virus (viremia), and viral DNA (leukoDNAemia). Because HCMV does not fully replicate in PMN, it is generally hypothesized that virions and viral materials are taken up by phagocytosis from fully permissive HCMV-infected endothelial cells. However, no experimental evidence has ever been provided for these PMN-endothelium interactions. PMN from 11 donors were cocultured with endothelial cells infected with an endothelium-adapted HCMV strain and with human fibroblasts infected with low-passaged clinical and laboratoryadapted HCMV strains. pp65-positive PMN were detected after coculture with both HCMV-infected endothelial and fibroblast cells, provided that wild and not laboratoryadapted strains were used. In addition, cocultured PMN carried infectious virus as demonstrated by virus isolation and presence of complete virus particles by electron microscopy. Moreover, high levels of viral DNA were consistently detected by quantitative PCR in cocultured PMN. Thus, we have generated in vitro the three most important viral parameters detected in patients with disseminated HCMV infection (antigenemia, viremia, and leukoDNAemia). The failure of laboratory-adapted HCMV strain to induce this phenomenon demonstrates that important modifications have occurred in attenuated viral strains affecting basic biological functions. (J. Clin. Invest. 1998. 101:2686-2692.) Key words: endothelial cells - fibroblasts - cocultivation - polymorphonuclear leukocytes • immunocompromised patients
\end{abstract}

\section{Introduction}

One of the most rapid approaches to the diagnosis and monitoring of systemic human cytomegalovirus (HCMV) ${ }^{1}$ infec-

Address correspondence to Maria Grazia Revello, Servizio di Virologia, IRCCS Policlinico San Matteo, 27100 Pavia, Italy. Phone: 39-382502634. FAX: 39-382-502599. E-mail: virology@ipv36.unipv.it

Received for publication 2 September 1997 and accepted in revised form 15 April 1998.

1. Abbreviations used in this paper: EC, endothelial cells; EM, electron microscope; HCMV, human cytomegalovirus; HELF, human embryonic lung fibroblasts; HUVEC, human umbilical vein endothelial cells; ICAM-1, intracellular adhesion molecule 1; LFA-1, lymphocyte function-associated antigen 1; p.i., post infection.

J. Clin. Invest.

(C) The American Society for Clinical Investigation, Inc. 0021-9738/98/06/2686/07 \$2.00

Volume 101, Number 12, June 1998, 2686-2692

http://www.jci.org tions is the detection of HCMV antigens in nuclei of peripheral blood leukocytes and, in particular, of PMN. This assay is known as the antigenemia test (1). The major HCMV antigen detected in this assay is the $65-\mathrm{kD}$ lower matrix phosphoprotein or pp65 (ppUL83; 2). In addition to pp65, PMN can carry infectious HCMV particles that can be recovered after cocultivation with human embryonic lung fibroblast (HELF) cells (3). Although early replicative events do seem to occur in PMN $(4,5)$, it is currently recognized that pp65 and infectious virus are mostly uptaken by phagocytosis. From a pathogenetic standpoint, it has been speculated that HCMV-infected leukocytes can contribute to spreading of HCMV infection by infecting vascular endothelial cells (EC; 6, 7). Conversely, HCMV-infected EC are thought to be the most likely source of both infectious virus and pp65 for PMN, although no evidence for such a phenomenon has ever been reported either in vivo or in vitro. In this report, we demonstrate that PMN can uptake HCMV pp65 and infectious virus in vitro after cocultivation with either virus-infected EC or HELF, provided that wild and not laboratory-adapted HCMV strains are used.

\section{Methods}

Cell cultures and virus strains. Human umbilical vein endothelial cells (HUVEC) were obtained by trypsin treatment of umbilical cord veins, propagated as reported (8), and used at passages 5-10. Culture medium for HUVEC was M199 supplemented with 20\% FCS, endothelial cell growth factor (Sigma Chemical Co., St. Louis, MO), and $5 \mathrm{U} / \mathrm{ml}$ sodium heparin. In addition, a HELF cell strain originally developed in our laboratory was used at passages 20-30. Culture medium for HELF was Earle's minimum essential medium-supplemented with $10 \%$ FCS in the growth medium and $2 \%$ FCS in the maintenance medium.

An HCMV strain (VR\#6110) recovered from blood of an AIDS patient was adapted to growth in HUVEC. After three initial passages in HELF cultures, VR\#6110 underwent 28 passages in HUVEC (VR\#6110/28 $8_{\mathrm{E}}$ ) before use. In addition, seven clinical HCMV isolates, including VR\#6110, were propagated in HELF cultures and used at passages 2-20. The seven HCMV strains were isolated from urine, amniotic fluid, human milk, lung biopsy, cervix, and buffy coat (two strains), respectively. HCMV laboratory strains AD169, Davis, and Towne (American Type Culture Collection, Rockville, MD) were propagated in our laboratory for several years. Cells and viral strains were routinely checked for mycoplasma contamination by Hoechst staining (9).

Isolation of PMN. Human PMN were obtained from seven HCMVseropositive and four HCMV-seronegative healthy donors. HCMV serostatus was determined by using a previously described ELISA assay (10). $1 \mathrm{ml}$ of $6 \%$ dextran solution (molecular weight 70,000 ) in saline was added to $5.0 \mathrm{ml}$ of heparinized blood. After incubation at $37^{\circ} \mathrm{C}$ for $\sim 30 \mathrm{~min}$, the supernatant was dispensed onto FicollHypaque and centrifuged at $600 \mathrm{~g}$ for $35 \mathrm{~min}$. Contaminating red blood cells were removed by hypotonic lysis $\left(1 \mathrm{ml} 0.8 \% \mathrm{NH}_{4} \mathrm{Cl}\right.$ for 2 min). The purity of harvested PMN was $>95 \%$, as assessed by May Grünwald-Giemsa as well as by chloro-acetate esterase staining. Cell 
viability was determined by trypan blue exclusion and was consistently $>95 \%$.

Cocultivation of PMN and HCMV-infected HUVEC or HELF. HCMV-infected HUVEC or HELF monolayers showing $>80 \%$ cytopathic effect were trypsinized. Infected cells were then mixed with uninfected HUVEC or HELF cells at 1:2 ratio and plated in 24-well cell culture plates $\left(10^{5}\right.$ cells/well $)$. After $72 \mathrm{~h}$, monolayers were washed and inoculated with $4 \times 10^{5} \mathrm{PMN}$. Cocultivation was continued for $24 \mathrm{~h}$ at $37^{\circ} \mathrm{C}$ unless otherwise indicated.

pp65 detection in PMN after cocultivation. PMN were recovered from HCMV-infected monolayers by gentle pipetting and aspiration of medium. Supernates were centrifuged at 1,200 rpm for $5 \mathrm{~min}$, and cell pellets were washed twice, counted, and suspended at a concentration of $2 \times 10^{6} / \mathrm{ml} .100 \mu l\left(2 \times 10^{5}\right.$ cells $)$ were then used for cytospin preparations that were fixed and stained using a previously reported procedure $(2,11)$. The number of pp65-positive PMN was counted and recorded. pp65-positive HUVEC or HELF cells were easily distinguished from pp65-positive PMN by size and by the predominant cytoplasmic staining, in contrast to the exclusive PMN nuclear staining. In addition, detection of pp65-positive PMN was also performed in cytopsin preparations of migrated or FACS ${ }^{\circledR}$-purified PMN, as described below.

In some experiments, PMN suspensions were incubated with $\mathrm{HCMV}$-infected HUVEC for $1 \mathrm{~h}$ at $37^{\circ} \mathrm{C}$. Supernatant containing unbound cells was then harvested and processed as described below. The EC monolayer was carefully washed to remove any residual, unbound PMN, refed with fresh medium, and cocultivation continued for $24 \mathrm{~h}$. At the end of the cocultivation time, supernatants containing de-adhered PMN were harvested and centrifuged. The adhering PMN were recovered by trypsin treatment of HCMV-infected HUVEC monolayers. Both cell fractions (adhering and nonadhering collected at 1- and 24-h coculture) were then washed twice, fixed, permeabilized, and stained for pp65 with specific mAb after a reported procedure for pp65 antigenemia determination (11). In parallel preparations, an irrelevant isotype-matched $\mathrm{mAb}$ was used. Samples were then analyzed on a FACScan ${ }^{\circledR}$ (Becton Dickinson, San Jose, CA) flow cytometer. The instrument setting was preliminarily carried out using isolated PMN and HUVEC to create gated cytograms corresponding to the physical parameters (forward scatter and side scatter) of each cell subset. Staining of the cocultured cells using a myeloid specific marker (anti-CD $18 \mathrm{mAb}$ TS.18, generously provided by T.A. Springer, Harvard University, Cambridge, MA) confirmed that $>95 \%$ of cells in the PMN gate and $<5 \%$ of cells in the HUVEC gate were of myeloid lineage.

PMN-mediated HCMV transmission and viral DNA detection in cocultured PMN. After cocultivation, to separate PMN from infected cells, $5 \times 10^{5}-10^{6} \mathrm{PMN}$ in $250 \mu \mathrm{l}$ RPMI 1640 (Flow Laboratories, Irvine, Scotland) supplemented with $1 \%$ FCS (GIBCO BRL, Life Technologies, Paisley, Scotland) were placed in the upper compartment of each cell culture insert $\left(5 \mu \mathrm{m}\right.$ pore size, $6.5 \mathrm{~mm}$ diameter Transwell ${ }^{\circledR}$; COSTAR, Cambridge, MA) and incubated for $3 \mathrm{~h}$ at $37^{\circ} \mathrm{C}$ in a $5 \%$ $\mathrm{CO}_{2}$ humidified atmosphere. The bottom compartment contained $10^{-8}$ M FMLP (Sigma Chemical Co., St. Louis, MO) in $500 \mu$ l RPMI 1640 plus $1 \%$ FCS (12). After incubation, the chambers were shaken to dislodge PMN from the lower surface of the inserts. PMN were then collected, extensively washed, and then inoculated $\left(2 \times 10^{5}\right.$ cells) onto HELF or HUVEC monolayers grown in shell vials. Early identification of infectious virus was then accomplished $16-24 \mathrm{~h}$ post infection (p.i.) by using the indirect immunofluorescence technique and a mAb to the HCMV major immediate early protein p72 (3). p72-positive nuclei were then counted and the number recorded. In parallel, HCMV DNA was quantitated in $10^{5} \mathrm{PMN}$ aliquots recovered after migration according to a reported PCR procedure using a primer pair relevant to exon 4 of the major immediate early gene (primer N1, nt 941-959; N2, nt 1431-1412) (13). In some experiments, PMN aliquots were treated with deoxyribonuclease (Boehringer Mannheim $\mathrm{GmbH}$, Mannheim, Germany) for $30 \mathrm{~min}$ before DNA extraction. In addition, $2 \times 10^{5}$ aliquots of migrated PMN were used for cytospin preparations for pp65 detection, as described above. Purity of migrated PMN was assessed by the May-Grünwald staining and was consistently $>95 \%$. Similarly, cell viability was $>95 \%$.

PMN purification by $F A C S^{\circledR}$. In some experiments, the migrated fraction of PMN was further purified by FACS $^{\circledR}$ after staining of the cells for CD 66b (Immunotech, Instrumentation Laboratory, Milan, Italy). Cell sorting was performed with a FACStar ${ }^{\circledR}$ PLUS (Becton Dickinson, Mountain View, CA). Laser output was $240 \mathrm{~mW}$ at 488 $\mathrm{nm}$. Sorting was carried out according to standard FACStar ${ }^{\circledR}$ specifications with a three-drop deflection criterion using a $70-\mu \mathrm{m}$ nozzle. The sorted PMN fraction was then used for quantification of pp65positive cells, infectious virus, and viral DNA. The same parameters were determined on aliquots of migrated PMN before FACS ${ }^{\circledR}$ purification.

Blocking experiments. PMN were first cultured into trans-well culture inserts of $0.4-\mu \mathrm{m}$ pore membrane (Becton Dickinson, Bedford, MA) that prevented PMN contact with the underlying cell monolayer. In addition, PMN were pretreated for $30 \mathrm{~min}$ with $6 \mu \mathrm{g} /$ $\mathrm{ml}$ of a mAb directed against the common beta chain (CD18) of lymphocyte function-associated antigen (LFA-1; DAKO, Glostrup, Denmark). Furthermore, HCMV-infected HUVEC or HELF monolayers were preincubated for $30 \mathrm{~min}$ with $15.2 \mu \mathrm{g} / \mathrm{ml}$ of an anti-intracellular adhesion molecule 1 (ICAM-1) mAb (DAKO). Cocultures were then carried out in the presence of either $\mathrm{mAb}$ or of an irrelevant, isotype-matched control $\mathrm{mAb}$.

Electron microscopy (EM). In EM studies, cocultured PMN were fixed with Karnovsky's fixative, fixed afterwards with $1.5 \% \mathrm{OsO}_{4}$ in $0.2 \mathrm{M}$ cacodylate buffer, $\mathrm{pH}$ 7.3, dehydrated, and embedded in EponAraldite resin either before or after purification. Ultrathin sections were stained with lead citrate and uranyl acetate, and examined with a Zeiss 902 electron microscope (Oberkochen, Germany).

\section{Results}

HCMV pp65 detection in cocultured PMN. In initial experiments, PMN obtained from one seronegative and one seropositive subject were either incubated with AD169 cell-free suspension or cocultured with AD169-infected HELF cells. pp65-specific staining was never observed in the nucleus of PMN.

However, when PMN from the same two subjects were cocultured with HUVEC infected with VR\#6110/28 , pp65 was readily detected in the nucleus of PMN from both individuals with the same staining pattern observed ex vivo in patients

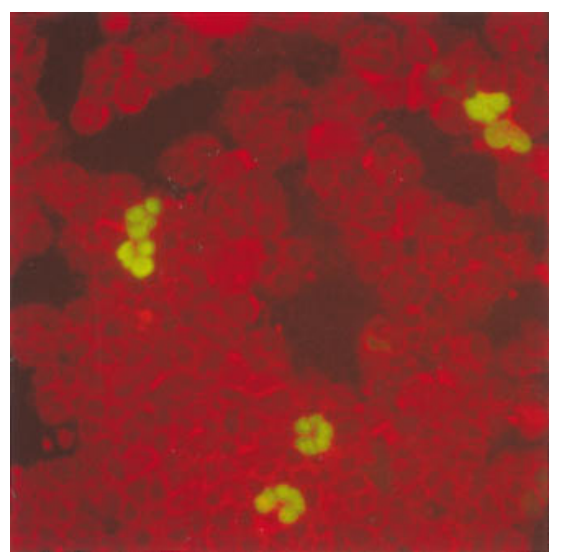

Figure 1. Immunofluorescent staining of HCMV pp65 in the nucleus of PMN recovered after a 24-h coculture with HUVEC infected with VR\#6110/28 . 
Table I. Relationship of Type of Cell Culture and HCMV Strains in Generating pp65-positive PMN

\begin{tabular}{|c|c|}
\hline PMN coculture condition & $\begin{array}{l}\text { pp65-positive } \\
\text { PMN }\end{array}$ \\
\hline VR\#6110/28 $8_{\mathrm{E}}$-infected HUVEC & present \\
\hline AD169-infected HELF + conditioned medium* & absent \\
\hline AD169-infected HELF + control medium* & absent \\
\hline VR\#6110/28 $8_{\mathrm{E}}$-infected HELF + conditioned medium & present \\
\hline VR\#6110/28 $8_{\mathrm{E}}$-infected HELF + control medium & present \\
\hline VR\#6110/4 (buffy coat)-infected HELF & present \\
\hline VR\#6333/4 (buffy coat)-infected HELF & present \\
\hline VR\#6347/2 $2_{\mathrm{F}}$ (urine)-infected HELF & present \\
\hline VR\#6348/2 F (amniotic fluid)-infected HELF & present \\
\hline VR\#6340/3 (human milk)-infected HELF & present \\
\hline VR\#6343/3 (lung biopsy)-infected HELF & present \\
\hline VR\#1814/20 ${ }_{\mathrm{F}}$ (cervix)-infected HELF & present \\
\hline Towne-infected HELF & absent \\
\hline Davis-infected HELF & absent \\
\hline
\end{tabular}

* Clarified medium collected from VR\#6110/28 $8_{\mathrm{E}}$-infected (and mockinfected) HUVEC cell cultures.

with pp65 antigenemia (Fig. 1). To assess whether VR\#6110/ $28_{\mathrm{E}}$-infected HUVEC could release soluble factor(s) responsible for (mediating) pp65 uptake by PMN, cocultivation experiments (Table I) were performed using clarified supernatants collected from VR\#6110/28 $8_{\mathrm{E}}$-infected (and mock-infected) HUVEC cultures as conditioned (and control) medium. Cocultivation of PMN and AD169-infected HELF in the presence of conditioned medium did not result in the detection of any pp65-positive PMN, whereas pp65-positive PMN could be detected after cocultivation with HELF cultures infected with cell-free VR\#6110/28 $8_{\mathrm{E}}$ both in the presence of conditioned and control medium (Table I). These results also demonstrated that fibroblasts infected with VR\#6110/28 E $_{\mathrm{E}}$ could efficiently induce pp65 uptake by PMN.

This raised the question of whether the phenomenon was strain-specific (VR\#6110/28 E) or extended to any clinical strain. Results reported in Table I show that pp65-positive PMN could be detected after cocultivation with HELF infected with any of seven clinical HCMV isolates at passage 2-20 (including an early passage of VR\#6110), but not with HELF infected with any of the laboratory-adapted strains (AD169, Towne, Davis).

Kinetics and frequency of pp65 uptake by PMN after cocultivation. PMN were examined for pp65 staining after a 1-, 6-, 24-, 48-, 72-, and 96-h cocultivation with either HUVEC or HELF VR\#6110/28 $8_{\mathrm{E}}$-infected monolayers. In a small number of PMN (1-2\% of the peak value), pp65 was detected as early as after $1 \mathrm{~h}$ (Fig. $2 A$ ). Number of pp65-positive cells increased in time reaching a peak after a 48 -h coculture.

As for PMN nuclear morphology, $>90 \%$ pp65-positive PMN recovered after a 24-h coculture had $<4$ lobes, whereas at later times frequency of pp65-positive PMN exhibiting a multilobed nucleus (5-10 lobes) slightly increased. Based on this observation and the additional finding that PMN recovered after a 48-h coculture showed reduced migration capacity (see below), a 24-h cocultivation was chosen as the optimal time for standard experiments.
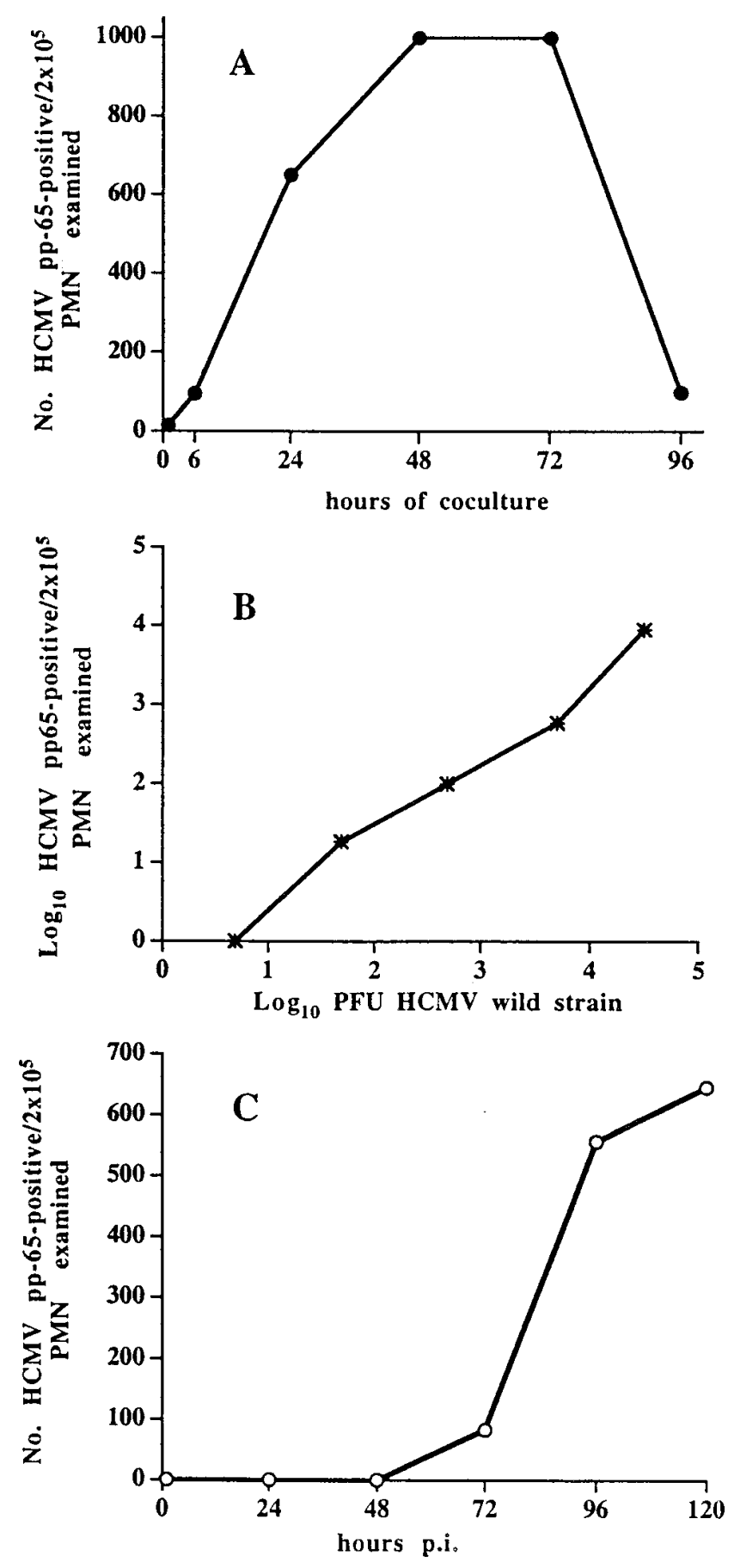

Figure 2. Number of pp65-positive $/ 2 \times 10^{5} \mathrm{PMN}$ at: $(A)$ different times of cocultivation with VR\#6110/28 $8_{\mathrm{E}}$-infected HUVEC; $(B)$ after a 24-h coculture with HELF infected with serial dilutions of clinical strain VR\#6347/2 $;$; C ) after a 3-h coculture with VR\#6347/2 $\mathrm{F}^{-}$ infected HELF at different times p.i.

Frequency of pp65-positive PMN varied from 0.2 to $5 \%$ and appeared directly related to the degree of infection of the cell monolayer. In fact, when PMN were cocultured for $24 \mathrm{~h}$ with HELF cells infected for $96 \mathrm{~h}$ with $\log _{10}$ dilutions of a lowpassage cell-free HCMV strain (VR\#6347/2 F), a progressive increase of pp65-positive PMN was observed in parallel with increasing amounts of input virus (Fig. $2 B$ ). 
To investigate pp65 uptake in relation to the time-course of HCMV infection, HELF monolayers were infected with $10^{-1}$ dilution of VR\#6347/2 . PMN were then added to monolayers 1, 24, 48, 72, 96, and $120 \mathrm{~h}$ p.i., and cocultivated for $3 \mathrm{~h}$. As shown in Fig. $2 C$, pp65-positive PMN were not detected until $72 \mathrm{~h}$, and then their relative number increased up to $120 \mathrm{~h}$ p.i.

$P M N$-mediated transmission of infectious virus and detection of viral DNA in cocultered PMN. The unequivocal demonstration that PMN cocultured with HCMV-infected cells did not only express pp65, but could also transmit infectious virus, required removal of all HCMV-infected cells (either HUVEC or HELF) present in the PMN suspension recovered after cocultivation. This was achieved by taking advantage of the capacity of PMN to migrate through natural and artificial barriers in response to chemoattractants. Control experiments indicated that neither HUVEC nor HELF (infected or mock infected) were able to migrate in response to FMLP. On the other hand, $30-50 \%$ of PMN recovered after a $24-\mathrm{h}$ coculture with HCMV-infected or mock-infected HUVEC or HELF monolayers, consistently migrated in response to FMLP, whereas PMN recovered after 48- and 72-h cocultures showed decreasing migrating capacity from 20 to $\sim 1 \%$, respectively. In addition, PMN positive for pp65 appeared to migrate as efficiently as pp65-negative PMN as indicated by the comparable relative proportion of positive cells before and after migration. Infectious virus was recovered from PMN of six subjects (two seronegative and four seropositive) after a 24-h cocultivation with either HUVEC or HELF-infected cells. HCMV was isolated both on HUVEC and HELF monolayers in comparable amounts. Mean number of p72-positive cells detected by the shell vial assay was 49.4 (range $2-300$ ) $/ 2 \times 10^{5} \mathrm{PMN}$ inoculated. On the other hand, supernatants from the last washing, which were inoculated in parallel with migrated PMN, were negative for virus isolation.

Viral DNA was detected in each aliquot of migrated PMN with a mean number of genome equivalents of $3 \times 10^{5}$ (range $\left.10^{5}-10^{6}\right) / 10^{5}$ PMN. The amount of viral DNA did not significantly change after deoxyribonuclease treatment before extraction (data not shown).

In some experiments, migrated PMN were further purified by $\mathrm{FACS}^{\circledR}$ and sorted population examined for presence of pp65, infectious virus, and DNA. The same parameters were determined before FACS ${ }^{\circledR}$ purification. Results of a representative experiment are reported in Table II, which shows that grossly comparable levels of antigenemia and leukoDNAemia

Table II. Results of Viral Parameters Determined on Migrated PMN Fractions before and after FACS ${ }^{\circledR}$ Purification

\begin{tabular}{lccc}
\hline & \multicolumn{3}{c}{ Viral parameter } \\
\cline { 2 - 4 } \multicolumn{1}{c}{ PMN fraction following } & pp65* & Infectious virus/DNA & DNA $^{\S}$ \\
\hline $\begin{array}{l}\text { Migration to FMLP } \\
\text { Migration to FMLP and } \\
\text { FACS }^{\circledR} \text { purification }\end{array}$ & $2 \times 10^{3}$ & 49 & $5 \times 10^{5}$ \\
& $2 \times 10^{3}$ & 7 & $\geq 10^{6}$
\end{tabular}

*No. of HCMV pp65-positive PMN/2 $\times 10^{5}$ examined (pp65 antigenemia). ${ }^{\ddagger}$ No. of HCMV p72-positive HELF nuclei/2 $\times 10^{5} \mathrm{PMN}$ inoculated (viremia). ${ }^{\S}$ No. of HCMV genome equivalents $/ 10^{5} \mathrm{PMN}$ examined (leukoDNAemia).
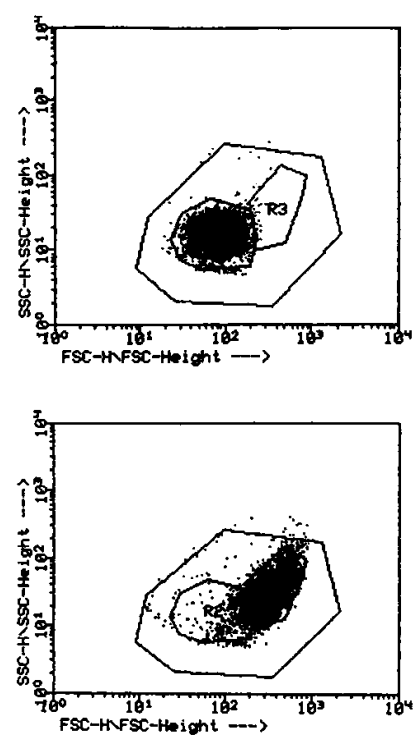

Figure 3. FACScan ${ }^{\circledR}$ gated cytograms, based on physical parameters (x axis, FSC; y axis, $S S C)$ for the identification of PMN in cocultures with

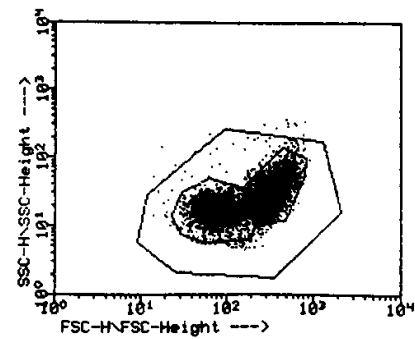
HCMV-infected HUVEC. PMN (top) have well defined FSC and SSC values that do not overlap with HUVEC ( $m i d$ dle). Cocultured cells can be assigned to either population on the basis of their inclusion in preset gates created as described (see Methods section).

were detected in migrated PMN before and after FACS ${ }^{\circledR}$ purification, whereas, for viremia, levels of infectious virus were slightly decreasing.

Contact-dependent uptake of pp65 by PMN cocultured with $H C M V$-infected HUVEC. To assess whether intercellular contact was required for pp65 uptake by PMN from HCMVinfected EC monolayers, we used three independent approaches. First, no pp65-positive PMN was detected after a 24-h coculture, when contact with HCMV-infected HUVEC or HELF monolayers ( $96 \mathrm{~h}$ p.i.) was prevented by trans-well inserts. Second, anti-LFA-1 (CD18) antibody decreased the number of pp65-positive PMN from $1486 \pm 1166$ to $216 \pm 159.9$ (85.46\% reduction). In addition, the $\mathrm{mAb}$ anti-ICAM-1 reduced the number of pp65-positive PMN from $1607 \pm 1323.3$ to $365 \pm 256.7$ (77.28\% reduction). However, whereas the level of the blocking effect observed with CD18 mAb was more consistent (66.6-93\% reduction), that of anti-ICAM-1 was more variable (25-92.5\% reduction). Finally, after a 1-h coculture with infected EC, nonadherent PMN were carefully and extensively removed by repeated washings of the coculture, followed by coculturing of the adherent fraction of PMN for an additional $24 \mathrm{~h}$. To identify PMN, we used a flow cytometry approach with a predetermined setting for the separation of PMN from EC based on physical parameters (Fig. 3). The results of these experiments are reported in Table III, which shows that: (a) the percentage of pp65-positive cells dramatically increased with time in the adhering fraction of PMN, and (b) pp65-positive PMN recovered in suspension after a 24-h coculture represent PMN initially adhering to and subse- 
Table III. Flow Cytometry Analysis of HCMV pp65 in Adhering and Nonadhering Cocultured PMN*

\begin{tabular}{llr}
\hline & \multicolumn{2}{c}{$\%$ pp65-positive PMN after time of coculture } \\
\cline { 2 - 3 } PMN fraction & $1 \mathrm{~h}$ & $24 \mathrm{~h}$ \\
\hline Adhering & 5.70 & 80.45 \\
Nonadhering & 1.25 & 65.25 \\
\hline
\end{tabular}

* Reported data are representative of three different experiments.

quently detaching from HCMV-infected EC. Thus, transient adhesion of PMN to HCMV-infected EC does occur and appears essential for pp65 uptake.

$E M$. Complete (enveloped) virus particles were detected by EM in the cytoplasm of some PMN after a 24-h coculture (Fig. 4). However, more often, naked particles were observed either within vacuoles or not (not shown). In addition, phagocytosis of dense bodies as well as presence of dense bodies inside cytoplasmic vacuoles of PMN was often observed (Fig. 5).

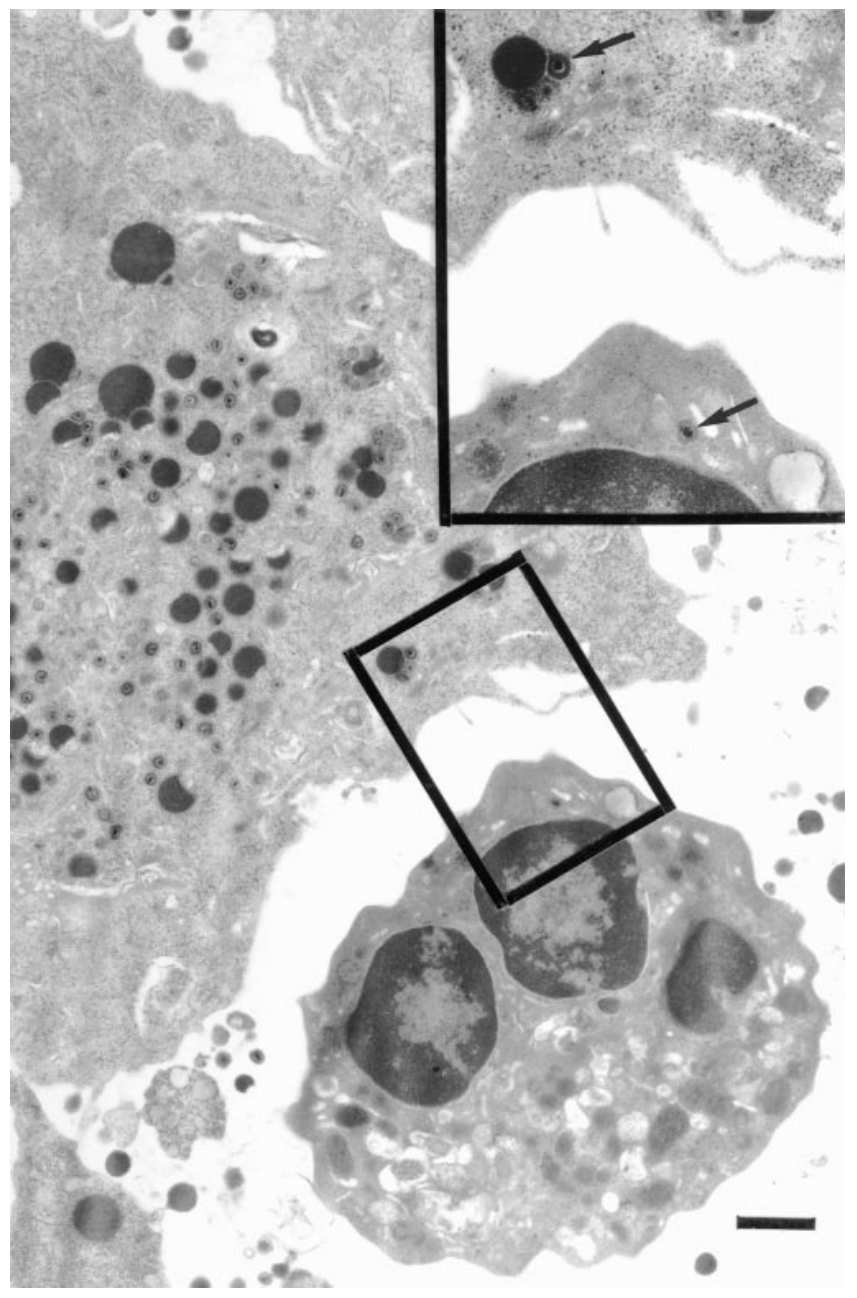

Figure 4. Ultrastructure of a PMN (right) and a portion of an HELF cell (left) after cocultivation. The insert shows enveloped virus particles (arrows) in both cells. Bar, $0.8 \mu \mathrm{m}$.

\section{Discussion}

The most important findings reported in the present study are the following: (a) pp65 is detected in PMN cocultured in vitro with EC infected with a HCMV clinical strain recovered from blood and propagated in EC; (b) pp65 uptake does also occur when PMN are cocultured with HELF cells infected with lowpassage HCMV isolates recovered from different body sites; (c) the phenomenon was never observed with PMN cocultured with HELF infected with laboratory-adapted AD169, Davis, and Towne HCMV strains or with PMN incubated with cellfree suspension of either AD169 or low-passage clinical strains; (d) PMN cocultured with HCMV-infected HUVEC or HELF cells are capable of transmitting infectious virus when cocultured with fresh HUVEC or HELF cells; (e) cocultured PMN carry high levels of viral DNA. Optimal time for PMN
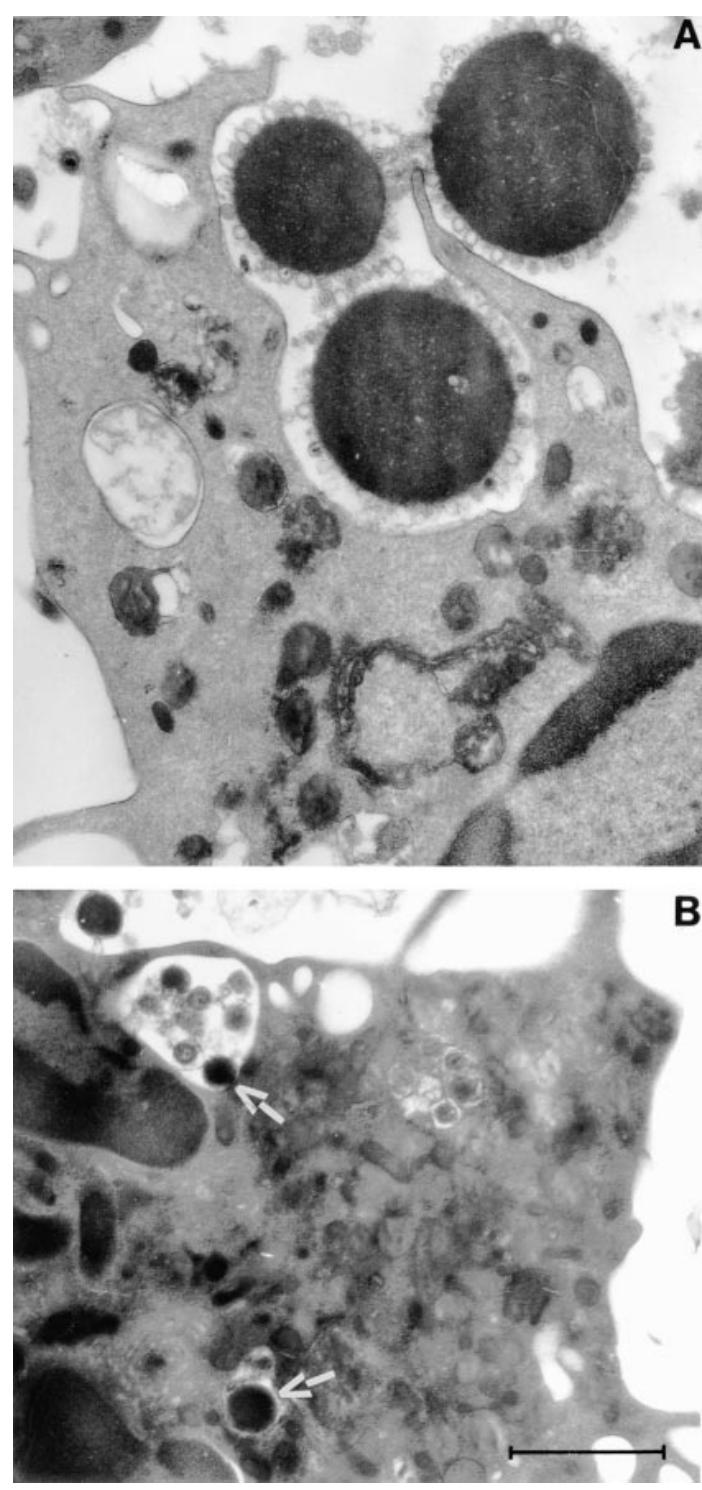

Figure 5. Multiple dense bodies are either $(A)$ in the process of being taken up or $(B)$ are present in the cytoplasm of a PMN (white arrows). Bar, $1.1 \mu \mathrm{m}$. 
cocultivation is $24 \mathrm{~h}$ because after a 48-96-h cocultivation the number of viable and migrating PMN progressively decreases.

Our data provide evidence that in vivo and in vitro PMNEC interactions may be similar as suggested by the following events: (a) upon adhesion to HCMV-infected EC, a low percentage of PMN $(0.2-5 \%$, a frequency comparable to that observed in vivo in patients with the highest antigenemia levels) acquire pp65 that localizes in the nucleus, as observed in ex vivo cytospin preparations; (b) pp65-positive PMN detach from infected EC and can be recovered in coculture medium (corresponding to the in vivo pp65 antigenemia detected in circulating PMN); (c) PMN can take up and transmit infectious virus from infected to uninfected EC or HELF (corresponding to the in vivo viremia); (d) cocultured PMN carry viral DNA (corresponding to the in vivo leukoDNAemia). In addition, during cocultivation, infected HUVEC may detach from the cell monolayer and be released together with cocultured PMN in the medium. This event perfectly mimics what has been observed in vivo in patients with disseminated HCMV infection and organ involvement, in whom circulating HCMVinfected EC become detectable in buffy coat samples examined for pp65 antigenemia $(6,7)$.

It has been reported that PMN adherence to HCMVinfected EC significantly increases by $24 \mathrm{~h}$ p.i. and is not induced by soluble components released in the medium by virusinfected cells $(14,15)$. More recently, Sedmak et al. showed that HCMV infection substantially upregulates the expression of the adhesion molecule ICAM-1 on infected EC (16), and the same appears to occur in HCMV-infected HELF (17). Thus, major interactions between HCMV-infected EC (or HELF) and PMN would involve this molecule and its known ligands, LFA-1 and MAC-1, which belong to the CD11/18 integrin subfamily and are predominantly found on the surface of PMN (12). In agreement with these data, our results indicate that adherence of PMN to infected monolayers does occur and is required for uptake of pp65. In fact, when cell to cell contact was physically (membrane insert) or immunologically (anti-CD18, anti-ICAM-1) prevented, pp65-positive PMN were absent or greatly reduced in number, respectively. Moreover, we showed that uptake of pp65 increases dramatically in PMN initially adhering to and subsequently detaching from $\mathrm{HCMV}$-infected EC. Contact or adherence to HCMV-infected EC has also been shown to be necessary for monocyte infection (18).

The most intriguing aspect of our study is the dramatic difference observed between HCMV laboratory-adapted strains and low-passaged clinical strains with respect to pp65 and infectious virus uptake by PMN. AD169 and Towne were developed as vaccine candidates and were serially passaged in HELF with the intent of attenuating their virulence before being used in clinical trials $(19,20)$. The ability of highly passaged laboratory strains to infect and replicate in EC has been reported to be markedly impaired for AD169 and Towne (21, 22), and absent for Davis (23). Moreover, 20 passages of a clinical HCMV strain on HELF were sufficient to cause definite alterations in its cytopathogenicity and replicative capacity in EC (21). Our own experience supports the above reported findings, in that all laboratory strains examined in the present study grew very poorly or not at all in EC.

Moreover, dramatic interstrain differences have been reported between attenuated (Towne and AD169) and a lowpassage (Toledo) strain of HCMV, regarding the ability to rep- licate in thymic stromal cells in SCID-hu mice (24), thus confirming that viral genetic determinants for tissue tropism may be lost during extensive propagation in cell cultures. Genetic analysis of attenuated AD169 and Towne, and the virulent strain Toledo demonstrated that a large DNA region (13$15 \mathrm{kbp}$ ) is missing in laboratory strains, whereas the additional DNA sequence of Toledo was conserved in five additional clinical isolates (25). The in vitro model herein described for generation of pp65 antigenemia unequivocally demonstrates that important biological modifications have occurred in attenuated strains resulting in the definite loss of the capacity to induce a basic pathogenetic phenomenon commonly occurring in vivo during disseminated HCMV infections of immunocompromised patients.

Finally, our results demonstrate that PMN upon coculture with HCMV-infected cells (either HUVEC or HELF) do uptake virions and can transmit infectious virus when cocultured with susceptible cells. This finding parallels the in vivo condition, where PMN do carry infectious virus providing the basis for the viremia assay (3). These observations indicate that virus infectivity must be somehow preserved inside PMN, as these cells do not support a complete virus replication cycle (4, 5). Indeed, we have detected by EM, complete, as well as more numerous incomplete virus particles and dense bodies in cytoplasmic vacuoles of cocultured PMN. Moreover, in agreement with ex vivo observations, cocultured PMN have been shown to carry viral DNA (leukoDNAemia; 13). However, in keeping with numerous in vivo reported findings $(13,26)$, levels of infectious virus (viremia) recovered are rather low compared to the amount of viral DNA (leukoDNAemia) detected. The various reasons for this may include degradation of uptaken virions during cocultivation and/or migration steps as well as uptake by PMN of free DNA from cell debris.

In conclusion, we have developed a model, which generates in vitro the three most important viral parameters detected in PMN of patients with disseminated HCMV infection, i.e., pp65 antigenemia, viremia, and leukoDNAemia. This model may represent the basis for future studies on the pathogenesis of HCMV infection in the immunocompromised host.

\section{Acknowledgments}

We thank Alberto Mantovani for helpful discussion, Sergio Bernasconi for FACS ${ }^{\circledR}$ purification, and Linda D'Arrigo for revision of the English.

This study was supported by Ministero della Sanità, Istituto Superiore di Sanità, IX and X Progetto Nazionale AIDS, and Ricerca Finalizzata IRCCS Policlinico San Matteo (grant 820RFM95/01).

\section{References}

1. Van der Bij, W., R. Torensma, W. Van Son, J. Anema, J. Schirm, A.M. Tegzess, and T.H. The. 1998. Rapid immunodiagnosis of active cytomegalovirus infection by monoclonal antibody staining of blood leukocytes. J. Med. Virol. 25:179-188.

2. Revello, M.G., E. Percivalle, A. Di Matteo, F. Morini, and G. Gerna. 1992. Nuclear expression of the lower matrix protein of human cytomegalovirus in peripheral blood leukocytes of immunocompromised viraemic patients. $J$. Gen. Virol. 73:437-442.

3. Gerna, G., M.G. Revello, E. Percivalle, M. Zavattoni, M. Parea, and M. Battaglia. 1990. Quantification of human cytomegalovirus viremia by using monoclonal antibodies to different viral proteins. J. Clin. Microbiol. 28:26812688.

4. Turtinen, L.W., R. Saltzman, M.C. Jordan, and A.T. Haasse. 1987. Interaction of human cytomegalovirus with leukocytes: in vivo analysis by in situ hybridization. Microb. Pathog. 3:287-297. 
5. Gerna, G., D. Zipeto, E. Percivalle, M. Parea, M.G. Revello, R. Maccario, G. Peri, and G. Milanesi. 1992. Human cytomegalovirus infection of the major leukocyte subpopulations and evidence for initial viral replication in polymorphonuclear leukocytes from viremic patients. J. Infect. Dis. 166:12361244.

6. Percivalle, E., M.G. Revello, L. Vago, F. Morini, and G. Gerna. 1993. Circulating endothelial giant cells permissive for human cytomegalovirus (HCMV) are detected in disseminated HCMV infections with organ involvement. J. Clin. Invest. 92:663-670.

7. Grefte, A., M. van der Giessen, W. van Son, and T.H. The. 1993. Circulating cytomegalovirus (CMV)-infected endothelial cells in patients with an active CMV infection. J. Infect. Dis. 167:270-277.

8. Vaccaro, P.S., L.B. Joseph, L. Titterington, and R.E. Stephens. 1987. Methods for the initiation and maintenance of human endothelial cell culture. Vasc. Surg. 21:391-400.

9. Chen, T.R. 1977. In situ detection of mycoplasma contamination in cell cultures by fluorescent Hoechst 33258 stain. Exp. Cell Res. 104:255-262.

10. Gerna, G., M.G. Revello, M. Palla, E. Percivalle, and M. Torsellini. 1992. Microneutralization as a reference method for selection of the cut-off of an enzyme-linked immunosorbent assay for detection of IgG antibody to human cytomegalovirus. Microbiologica (Pavia). 15:177-182.

11. Gerna, G., M.G. Revello, E. Percivalle, and F. Morini. 1992. Comparison of different immunostaining techniques and monoclonal antibodies to the lower matrix phosphoprotein (pp65) for optimal quantitation of human cytomegalovirus antigenemia. J. Clin. Microbiol. 30:1232-1237.

12. Springer, T.A. 1994. Traffic signals for lymphocyte recirculation and leukocyte emigration: the multistep paradigm. Cell. 76:301-314.

13. Gerna, G., F. Baldanti, A. Sarasini, M. Furione, E. Percivalle, M.G. Revello, D. Zipeto, and D. Zella. 1994. Effect of foscarnet induction treatment on quantitation of human cytomegalovirus (HCMV) DNA in peripheral blood polymorphonuclear leukocytes and aqueous humor of AIDS patients with HCMV retinitis. The Italian Foscarnet Study Group. Antimicrob. Agents Chemother. 38:38-44.

14. Span, A.H.M., C.P.A. Van Boven, and C.A. Bruggeman. 1989. The effect of cytomegalovirus infection on the adherence of polymorphonuclear leukocytes to endothelial cells. Eur. J. Clin. Invest. 19:542-548.
15. Span, A.H.M., M.C.E. Van Dam-Mieras, W. Mullers, J. Endert, A.D. Muller, and C.A. Bruggeman. 1991. The effect of virus infection on the adherence of leukocytes or platelets to endothelial cells. Eur. J. Clin. Invest. 21:331338

16. Sedmak, D.D., D.A. Knight, N.C. Vook, and J.W. Waldman. 1994. Divergent patterns of ELAM-1, ICAM-1, and VCAM-1 expression on cytomegalovirus-infected endothelial cells. Tranplantation. 12:1379-1385.

17. Grundy, J.E., and K.L. Downes. 1993. Up-regulation of LFA-3 and ICAM-1 on the surface of fibroblasts infected with cytomegalovirus. Immunology. 78:405-412.

18. Waldman, W.J., D.A. Knight, E.H. Huang, and D.D. Sedmak. 1995. Bidirectional transmission of infectious cytomegalovirus between monocytes and vascular endothelial cells: an in vitro model. J. Infect. Dis. 171:263-272.

19. Elek, S.D., and H. Stern. 1974. Development of a vaccine against mental retardation caused by cytomegalovirus infection in utero. Lancet. 1:1-5.

20. Plotkin, S.A., J. Farquhar, and E. Hornberger. 1976. Clinical trials of immunization with the Towne 125 strain of cytomegalovirus. J. Infect. Dis. 134 $470-475$.

21. Waldman, W.J., W.H. Roberts, D.H. Davis, M.V. Williams, D.D. Sedmak, and R.E. Stephens. 1991. Preservation of natural endothelial cytopathogenicity of cytomegalovirus by propagation in endothelial cells. Arch. Virol. 117: 143-164.

22. Radsak, K., R. Fuhrmann, R.P. Franke, D. Schneider, A. Kollert, K.H. Brucher, and D. Drenckhahan. 1989. Induction by sodium butyrate of cytomegalovirus replication in human endothelial cells. Arch. Virol. 107:151-158.

23. Friedman, H.M., E.J. Makarak, R.R. MacGregor, J. Wolfe, and N.A. Kefalides. 1981. Virus infection of endothelial cells. J. Infect. Dis. 143:266-273.

24. Brown, J.M., H. Kaneshima, and E.S. Mocarski. 1995. Dramatic interstrains differences in the replication of human cytomegalovirus in SCID-hu mice. J. Infect. Dis. 171:1599-1603.

25. Cha, T.-H., E. Tom, G.W. Kemble, G.M. Duke, E.S. Mocarski, and R.S. Spaete. 1996. Human cytomegalovirus clinical isolates carry at least 19 genes not found in laboratory strains. J. Virol. 70:78-83.

26. Gerna, G., M. Furione, F. Baldanti, and A. Sarasini. 1994. Comparative quantitation of human cytomegalovirus DNA in blood leukocytes and plasma of transplant and AIDS patients. J. Clin. Microbiol. 32:2709-2717. 